\title{
Use of the TI-700 cassette terminal in the INTERACT system
}

\author{
JAMES D. BELLUZZI \\ Psychopharmacology Department, Wyeth Laboratories, Inc., Philadelphia, Pennsylvania 19101
}

\begin{abstract}
The TI-700 terminal provides magnetic tape capability to the INTERACT user. The terminal is faster, quicker, and more versatile than a standard ASR-33 Teletype. Program duplication, editing, and storage is simplified and on-line program control of terminal operation is possible.
\end{abstract}

The recent addition of the Texas Instruments Silent 700 terminal to the peripherals compatible with the BRS/LVE INTERACT computer control system provides the user with a more flexible and powerful system. The purpose of the present paper is to acquaint the potential user with the capabilities and problems of the TI-700 terminal.

The system we use at Wyeth Laboratories employs a Data General Nova $2 / 10$ computer with $32 \mathrm{~K}$ words of core, eight 11-bit INTERACT stations, two TI-700 terminals with dual cassette drives, and an ASR-33 Teletype. Although the INTERACT system could run using a single terminal, we have configured ours to use one TI.700 as the input terminal, with parallel outputs to the other TI-700 and the Teletype. This configuration provides maximum flexibility and minimum interference between input and output functions. The Teletype is used to print data on pressure-sensitive labels that can be pasted directly into data books. In addition, it provides back-up paper-tape capability.

Installation of the TI-700 terminal is relatively simple. Five IC chips and a relay must be added to boards in the CPU and cassette unit to provide needed modification to the playback control, carriage return delay, and high-speed option.

\section{SPECIFICATIONS}

The printing mechanism consists of a 5 by 7 dot matrix that is electronically heated to form characters on heat-sensitive paper. This has the advantage of relatively fast ( 30 characters/sec, cps) and silent printing, but some might consider the heat-sensitive paper a disadvantage. The paper has worked well in our lab, but we do not use it for data storage, since our Teletype prints most of the data that is stored. The tape cassette drives consist of two complete record/playback units. These cassette units are independent except that they cannot be operated in parallel, i.e., if one is in the record mode, the other is in the playback mode. On-line data transfer rates of 30 to $120 \mathrm{cps}$ are available, while off-line rates as high as $326 \mathrm{cps}$ are available in certain modes. Our terminals also have two options, automatic search control and remote device control. The automatic search control enables the user to locate any data on the tape. The remote device control provides the capability of program control of output terminal functions. These features are described in more detail below. The cassette units record and play back in a block format. Data is entered into an 86-character buffer, and recording of the whole buffer takes place at one time. In the off-line mode, data in the buffer can be deleted, replaced, or changed through the keyboard.

\section{OPERATION}

The TI-700 terminals operate basically the same as a Teletype, with the cassette units replacing the papertape reader and punch. When the input terminal is on-line, data can be read in from the keyboard at $30 \mathrm{cps}$ (300 baud) or from the cassette unit at either 30 or 120 cps. With the dual cassette units, it is possible to read programs in from a previously prepared tape, get simultaneous printout on paper, and record the CPU output on the second cassette. However, this can be done only at $30 \mathrm{cps}$. If programs are input at $120 \mathrm{cps,}$ no printout is possible. At $120 \mathrm{cps}$ and with printout suppressed (CTRL-B), the input rate is approximately 300 ACT-III octal program blocks per minute. Once the cassette input is initiated, it is under CPU control, so it is not possible to edit tapes on-line.

The on-line operation of the output terminal provides data output to the printer and/or cassette unit. With the two-terminal configuration, the input and output are totally independent. The remote device control expands the capability of the output terminal by putting data output under program control so that the program can direct all operations of the terminal, such as cassette selection, record, rewind, print, playback, and automatic search functions. These various controls are issued by putting the appropriate two-character function code into print literals in the program, e.g., S1 [PRINT "DLE-3"] will load cassette 1. Using these control codes, the program can select the cassette, rewind it, load it, search for a particular place on the tape, and 
record the appropriate data. We used this capability to produce on-line data output tapes where each new piece of data is identified according to animal and treatment conditions and then added to the end of the appropriate group on the tape. At the end of the experiment, we can print this tape to produce a summary data sheet in which the data from each animal appear in the proper treatment group. This is very helpful in organizing the data, but there are some limitations. Since the output terminal cannot read information off the tape back to the CPU, there is no feedback to identify completion of a particular step. Thus, all operations must be properly timed so that no command is issued while the previous operation is still in progress. Also, data outputs from any other station will interfere with the execution of program control, so outputs of the whole system must be nonoverlapping.

Perhaps the most valuable feature of the TI-700 terminal is its powerful off-line editing capability. For most functions, it is significantly faster and more efficient than paper-tape systems. Tape duplicating can be done at $250 \mathrm{cps}$. Editing can be easily performed during duplication by use of the automatic search control. To add, change, or delete any part of the tape, the automatic search is activated and up to 16 characters of the data to be altered are entered as the search ID. When the search is started, everything on the tape is copied onto a second tape during the search. When the data in the ID are reached on the tape, the terminal responds with a line feed and a carriage return. All data in the 86-character buffer are printed out and can then be erased or added to before recording further. This process can be repeated any number of times, so that the entire tape can be edited and re-recorded during a single run through the original tape. This provides a quick and relatively simple means of making alterations to existing programs. However, certain precautions are necessary. When material is changed off-line, it is necessary to be familiar with the nonprinting control characters used in the ACT language. If these are not properly placed in new material or deleted from old material, the tape will not run when it is loaded into a station. The main difficulty with the editing is that it is complicated at first and, if care is not taken, valuable programs can be erased or overwritten. We routinely keep duplicates of all programs.

\section{SUMMARY}

The TI-700 terminal has performed well in our laboratory. Perhaps the biggest advantage of the terminal is the elimination of paper tapes. The cassettes are quite convenient to work with and have enough capacity to get all of the programs for our eight-station system on one side of a cassette. Programs can be retrieved using the automatic search control. We have not found the 120-cps input transfer rate to be limiting. We can load the computer and program all eight stations, which in our system uses almost $32 \mathrm{~K}$ of core, in less than $15 \mathrm{~min}$. In our configuration the terminals have provided trouble-free service for almost a year and their capabilities have been sufficient for our needs. 